BI-TP 2004/36

CERN-PH-TH/2004-236

HIP-2004-48/TH

hep-lat/0412008

\title{
Plaquette expectation value and gluon condensate in three dimensions
}

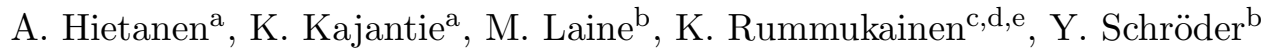 \\ a Theoretical Physics Division, Department of Physical Sciences, \\ P.O.Box 64, FI-00014 University of Helsinki, Finland \\ ${ }^{\mathrm{b}}$ Faculty of Physics, University of Bielefeld, D-33501 Bielefeld, Germany \\ ${ }^{\mathrm{c}}$ Department of Physics, Theory Division, CERN, CH-1211 Geneva 23, Switzerland \\ ${ }^{\mathrm{d} D e p a r t m e n t}$ of Physics, University of Oulu, P.O.Box 3000, FI-90014 Oulu, Finland \\ ${ }^{\mathrm{e}}$ Helsinki Institute of Physics, P.O.Box 64, FI-00014 University of Helsinki, Finland
}

\begin{abstract}
In three dimensions, the gluon condensate of pure SU(3) gauge theory has ultraviolet divergences up to 4-loop level only. By subtracting the corresponding terms from lattice measurements of the plaquette expectation value and extrapolating to the continuum limit, we extract the finite part of the gluon condensate in lattice regularization. Through a change of regularization scheme to $\overline{\mathrm{MS}}$ and (inverse) dimensional reduction, this result would determine the first non-perturbative coefficient in the weak-coupling expansion of hot QCD pressure.
\end{abstract}

December 2004 


\section{Introduction}

As non-Abelian gauge theories in three and four dimensions are confining, their properties need, in general, to be studied non-perturbatively. If the observables considered involve momenta or masses $(M)$ large compared with the confinement scale, however, then the conceptual framework of the operator product expansion [1 may allow to isolate the nonperturbative dynamics into only a few (gluon) condensates, while the rest of the answer can be computed by perturbative means. A classic example is the mass of a heavy quark-anti-quark bound state [2] The task faced by numerical lattice simulations might then get significantly simplified, as local condensates are simpler to measure with controlled systematic errors than correlation functions of heavy states.

On the other hand, the physical interpretation of a "bare" lattice measurement of a gluon condensate is non-trivial. The reason is that the condensate is represented by the expectation value of a dimensionful singlet operator and, in general, contains ultraviolet divergences of the same degree as its dimension. Operator product expansion type relations are often derived employing dimensional regularization, since the system then only contains one large parameter $(M)$ rather than two ( $M$ and the momentum cutoff), which simplifies the derivation considerably. Making use of lattice results in such a context requires then a transformation from lattice to continuum regularization. While in principle a well-defined perturbative problem (see, e.g., Refs. [3]), this is in practice somewhat problematic in four dimensions, given that there are contributions from all orders in the loop expansion.

The observable we consider in this paper is the (lowest-dimensional) singlet gluon condensate in three dimensions (3d), measured with pure SU(3) gauge theory. As 3d pure Yang-Mills theory is super-renormalisable, the problem of changing the regularization scheme becomes solvable: there are ultraviolet divergences up to 4-loop level only [4]. Furthermore, as we will elaborate in the following, all the divergences have been determined recently. These advances allow us to obtain a finite "subtracted" continuum value for the gluon condensate in lattice regularization. A conversion to the $\overline{\mathrm{MS}}$ scheme, amounting to the (perturbative) computation of the constant $c_{4}^{\prime}$ in Eq. (2.12) below, remains however a future challenge.

There might be various physics settings where the $3 \mathrm{~d}$ gluon condensate finds practical applications. The one that motivated us, is that this condensate appears in high-temperature physics, where the temperature $T$ plays the role of the mass scale $M$ mentioned above. Indeed $3 \mathrm{~d}$ pure Yang-Mills theory determines the leading non-perturbative contribution to the weakcoupling expansion of the pressure (and a number of other quantities) of physical QCD [5, 6], through a conceptual counterpart of the operator product expansion, called finite-temperature dimensional reduction [7, 8, 9]. Other applications might exist as well.

The plan of this paper is the following. In Sec. 2, we specify the observables considered and discuss the theoretical setting of our study. Numerical results from lattice Monte Carlo simulations are reported in Sec. 3, and we conclude in Sec. 4. 


\section{Theoretical setting}

We start this Section by formulating the observables that we are interested in, in the formal continuum limit of the theory. The ultraviolet (UV) divergences appearing in loop contributions are at this stage regulated through the use of dimensional regularization. Later on we go over to lattice regularization, in order to give a precise non-perturbative meaning to the observables introduced, allowing for their numerical determination.

The Euclidean continuum action of pure $\mathrm{SU}\left(N_{\mathrm{c}}\right)$ Yang-Mills theory can be written as

$$
S_{\mathrm{E}}=\int \mathrm{d}^{d} x \mathcal{L}_{\mathrm{E}}, \quad \mathcal{L}_{\mathrm{E}}=\frac{1}{2 g_{3}^{2}} \operatorname{Tr}\left[F_{k l}^{2}\right] .
$$

Here $d=3-2 \epsilon, g_{3}^{2}$ is the gauge coupling, $k, l=1, \ldots, d, F_{k l}=i\left[D_{k}, D_{l}\right], D_{k}=\partial_{k}-i A_{k}$, $A_{k}=A_{k}^{a} T^{a}, T^{a}$ are the Hermitean generators of $\mathrm{SU}\left(N_{\mathrm{c}}\right)$, normalised as $\operatorname{Tr}\left[T^{a} T^{b}\right]=\delta^{a b} / 2$, and repeated indices are assumed to be summed over. Leaving out for brevity gauge fixing and Faddeev-Popov terms, the "vacuum energy density" reads

$$
f_{\overline{\mathrm{MS}}} \equiv-\lim _{V \rightarrow \infty} \frac{1}{V} \ln \left[\int \mathcal{D} A_{k} \exp \left(-S_{\mathrm{E}}\right)\right]_{\overline{\mathrm{MS}}},
$$

where $V$ is the $d$-dimensional volume, $\mathcal{D} A_{k}$ a suitable (gauge-invariant) functional integration measure, and we have assumed the use of the $\overline{\mathrm{MS}}$ dimensional regularization scheme to remove any $1 / \epsilon$ poles from the expression. We note that $f_{\overline{\mathrm{MS}}}$ has the dimensionality $[\mathrm{GeV}]^{d}$.

In strict dimensional regularization, $f_{\overline{\mathrm{MS}}}$ of course vanishes order by order in the loop expansion, due to the absence of any mass scales in the propagators. This behaviour is unphysical, however, and due to an exact cancellation between UV and infrared (IR) divergences; for an explicit discussion at 3-loop level in a related case, see Appendix B of Ref. [10. In fact non-perturbatively the structure of $f_{\overline{\mathrm{MS}}}$ is rather

$$
f_{\overline{\mathrm{MS}}}=-g_{3}^{6} \frac{d_{A} N_{\mathrm{c}}^{3}}{(4 \pi)^{4}}\left[\left(\frac{43}{12}-\frac{157}{768} \pi^{2}\right) \ln \frac{\bar{\mu}}{2 N_{\mathrm{c}} g_{3}^{2}}+B_{\mathrm{G}}+\mathcal{O}(\epsilon)\right],
$$

where $d_{A} \equiv N_{\mathrm{c}}^{2}-1$, and we have introduced an $\overline{\mathrm{MS}}$ scheme scale parameter $\bar{\mu}$. The coefficient of the logarithm in Eq. (2.3) has been determined in Ref. [11] with a perturbative 4-loop computation, by regulating all the propagators by a small mass scale $m_{\mathrm{G}}$, and sending $m_{\mathrm{G}} \rightarrow$ 0 only after the computation (see also Ref. [12]). The non-perturbative constant part $B_{\mathrm{G}}{ }^{1}$ which actually is a function of $N_{\mathrm{c}}$, is what we would ultimately like to determine.

One direct physical application of $B_{\mathrm{G}}$ is that it determines the first non-perturbative contribution to the weak-coupling expansion of the pressure $p$ of QCD at high temperatures [5, 6]. To be precise, this contribution is of the form $\delta p=d_{A} N_{\mathrm{c}}^{3} g^{6} T^{4} B_{\mathrm{G}} /(4 \pi)^{4}$, where $g^{2}$ is the renormalised QCD gauge coupling. Terms up to order $\mathcal{O}\left(g^{6} \ln (1 / g)\right)$ are, in contrast, perturbative, and all known by now [12].

\footnotetext{
${ }^{1}$ In Ref. [12], $B_{\mathrm{G}}$ was denoted by $\beta_{\mathrm{G}}$, but we prefer to introduce a new notation here, in order to avoid confusion with the coupling constant $\beta$ appearing in Eq. (2.6). The subscript $\mathrm{G}$ might refer to gluons.
} 
For future reference, we note that given $f_{\overline{\mathrm{MS}}}$, we immediately obtain the gluon condensate:

$$
\begin{aligned}
\frac{1}{2 g_{3}^{2}}\left\langle\operatorname{Tr}\left[F_{k l}^{2}\right]\right\rangle_{\overline{\mathrm{MS}}} & \equiv-g_{3}^{2} \frac{\partial}{\partial g_{3}^{2}} f_{\overline{\mathrm{MS}}} \\
& =3 g_{3}^{6} \frac{d_{A} N_{\mathrm{c}}^{3}}{(4 \pi)^{4}}\left[\left(\frac{43}{12}-\frac{157}{768} \pi^{2}\right)\left(\ln \frac{\bar{\mu}}{2 N_{\mathrm{c}} g_{3}^{2}}-\frac{1}{3}\right)+B_{\mathrm{G}}+\mathcal{O}(\epsilon)\right] .
\end{aligned}
$$

We now go to the lattice. In the standard Wilson discretization, the lattice action, $S_{a}$, corresponding to Eq. (2.1), reads

$$
S_{a}=\beta \sum_{\mathbf{x}} \sum_{k<l}\left(1-\frac{1}{N_{\mathrm{c}}} \operatorname{Re} \operatorname{Tr}\left[P_{k l}(\mathbf{x})\right]\right),
$$

where $P_{k l}(\mathbf{x})=U_{k}(\mathbf{x}) U_{l}(\mathbf{x}+k) U_{k}^{-1}(\mathbf{x}+l) U_{l}^{-1}(\mathbf{x})$ is the plaquette, $U_{k}(\mathbf{x})$ is a link matrix, $\mathbf{x}+k \equiv \mathbf{x}+a \hat{\epsilon}_{k}$, where $a$ is the lattice spacing and $\hat{\epsilon}_{k}$ is a unit vector, and

$$
\beta \equiv \frac{2 N_{\mathrm{c}}}{g_{3}^{2} a}
$$

Note that the gauge coupling does not get renormalised in $3 \mathrm{~d}$, and the parameters $g_{3}^{2}$ appearing in Eqs. (2.1), (2.7) can hence be assumed finite and equivalent. The observable we consider is still the vacuum energy density, Eq. (2.2), which in lattice regularization reads

$$
f_{a} \equiv-\lim _{V \rightarrow \infty} \frac{1}{V} \ln \left[\int \mathcal{D} U_{k} \exp \left(-S_{a}\right)\right],
$$

where $\mathcal{D} U_{k}$ denotes integration over link matrices with the gauge-invariant Haar measure.

Now, being in principle physical quantities, the values of $f_{\overline{\mathrm{MS}}}$ and $f_{a}$ must agree, provided that suitable vacuum counterterms are added to the theory. Due to super-renormalizability, there can be such counterterms up to 4-loop level only [4], and correspondingly

$$
\begin{aligned}
\Delta f & \equiv f_{a}-f_{\overline{\mathrm{MS}}} \\
& =C_{1} \frac{1}{a^{3}}\left(\ln \frac{1}{a g_{3}^{2}}+C_{1}^{\prime}\right)+C_{2} \frac{g_{3}^{2}}{a^{2}}+C_{3} \frac{g_{3}^{4}}{a}+C_{4} g_{3}^{6}\left(\ln \frac{1}{a \bar{\mu}}+C_{4}^{\prime}\right)+\mathcal{O}\left(g_{3}^{8} a\right),
\end{aligned}
$$

where the $C_{i}$ are dimensionless functions of $N_{\mathrm{c}}$. The values of $C_{1}, C_{2}, C_{3}, C_{4}$ are known, as we will recall presently; $C_{1}^{\prime}$ is related to the precise normalisation of the Haar integration measure and void of physical significance; and $C_{4}^{\prime}$ is unknown as of today.

Correspondingly, the gluon condensates, i.e. the logarithmic derivatives of $f_{\overline{\mathrm{MS}}}, f_{a}$ with respect to $g_{3}^{2}$, can also be related by a perturbative 4-loop computation. Noting that threedimensional rotational and translational symmetries and the reality of $S_{a}$ allow us to write

$$
-g_{3}^{2} \frac{\partial}{\partial g_{3}^{2}} f_{a}=\frac{3 \beta}{a^{3}}\left\langle 1-\frac{1}{N_{\mathrm{c}}} \operatorname{Tr}\left[P_{12}\right]\right\rangle_{a},
$$


and employing Eqs. (2.5), 2.10), we obtain finally the master relation

$$
8 \frac{d_{A} N_{\mathrm{c}}^{6}}{(4 \pi)^{4}} B_{\mathrm{G}}=\lim _{\beta \rightarrow \infty} \beta^{4}\left\{\left\langle 1-\frac{1}{N_{\mathrm{c}}} \operatorname{Tr}\left[P_{12}\right]\right\rangle_{a}-\left[\frac{c_{1}}{\beta}+\frac{c_{2}}{\beta^{2}}+\frac{c_{3}}{\beta^{3}}+\frac{c_{4}}{\beta^{4}}\left(\ln \beta+c_{4}^{\prime}\right)\right]\right\} .
$$

The values of the constants $c_{1}, \ldots, c_{4}^{\prime}$ are trivially related to those of $C_{1}, \ldots, C_{4}^{\prime}$ in Eq. (2.10).

Now, a straightforward 1-loop computation yields

$$
c_{1}=\frac{d_{A}}{3} \approx 2.66666667,
$$

where the numerical value applies for $N_{\mathrm{c}}=3$.

The 2-loop term is already non-trivial: it was first computed in four dimensions in Ref. [13], and in three dimensions in Ref. [14. The $3 \mathrm{~d}$ result can be written in the form

$$
c_{2}=-\frac{2}{3} \frac{d_{A} N_{\mathrm{c}}^{2}}{(4 \pi)^{2}}\left(\frac{4 \pi^{2}}{3 N_{\mathrm{c}}^{2}}+\frac{\Sigma^{2}}{4}-\pi \Sigma-\frac{\pi^{2}}{2}+4 \kappa_{1}+\frac{2}{3} \kappa_{5}\right)=1.951315(2),
$$

where the coefficients $\Sigma, \kappa_{1}$ can be found in Refs. 4, 15, and we have defined

$$
\kappa_{5}=\frac{1}{\pi^{4}} \int_{-\pi / 2}^{\pi / 2} \mathrm{~d}^{3} x \mathrm{~d}^{3} y \frac{\sum_{i} \sin ^{2} x_{i} \sin ^{2}\left(x_{i}+y_{i}\right) \sin ^{2} y_{i}}{\sum_{i} \sin ^{2} x_{i} \sum_{i} \sin ^{2}\left(x_{i}+y_{i}\right) \sum_{i} \sin ^{2} y_{i}}=1.013041(1) .
$$

The numbers in parentheses in Eqs. (2.14), (2.15) indicate the uncertainties of the last digits.

The 3-loop term is well known in four dimensions since a long time ago [16, but the same computation has been carried out in three dimensions only very recently [17]:

$$
c_{3}=6.8612(2)
$$

This value improves on a previous estimate $c_{3}=6.90_{-0.12}^{+0.02}[18$, obtained through the evaluation of the 3-loop graphs with the method of stochastic perturbation theory [19].

The value of $c_{4}$ follows by a comparison of Eqs. (2.3) and (2.10): there is no $\bar{\mu}$-dependence in $f_{a}$, so that the one in $f_{\overline{\mathrm{MS}}}$ determines the coefficient of the logarithm in $\Delta f$. Consequently,

$$
c_{4}=8 \frac{d_{A} N_{\mathrm{c}}^{6}}{(4 \pi)^{4}}\left(\frac{43}{12}-\frac{157}{768} \pi^{2}\right) \approx 2.92942132 .
$$

The knowledge of $c_{1}, c_{2}, c_{3}, c_{4}$ allows us to subtract all the divergent contributions from the gluon condensate. A finite 4-loop term, parametrised by $c_{4}^{\prime}$ in Eq. (2.12), however still remains. It could in principle be determined by extending either the method of Ref. [17] or of Ref. 18 to 4-loop level. There is the additional complication, though, that intermediate steps of the computation require the use of an IR cutoff, which then cancels once the lattice and $\overline{\mathrm{MS}}$ results are subtracted, in Eq. (2.10). This computation has not been carried out yet, and therefore we will not be able to determine $B_{\mathrm{G}}$ in this paper. We can determine, however, the non-perturbative input needed for it (cf. Eq. (3.1) below), the purely perturbative determination of $c_{4}^{\prime}$ then remaining a future challenge. 


\begin{tabular}{ll}
\hline \hline$\beta$ & volumes \\
\hline \hline 12 & $24^{3}, 32^{3}, 48^{3}$ \\
16 & $24^{3}, 32^{3}, 48^{3}, 64^{3}$ \\
20 & $24^{3}, 32^{3}, 48^{3}$ \\
24 & $\left\{12^{3}, 14^{3}, 16^{3}, 20^{3}, 24^{3}\right\}, 32^{3}, 48^{3}, 64^{3}$ \\
32 & $\left\{14^{3}, 16^{3}, 20^{3}, 24^{3}, 32^{3}\right\}, 48^{3}, 64^{3}, 96^{3}$ \\
40 & $\left\{32^{3}\right\}, 48^{3}, 64^{3}, 96^{3}$ \\
50 & $\left\{20^{3}, 24^{3}, 26^{3}, 28^{3}, 32^{3}, 48^{3}\right\}, 64^{3}, 96^{3}, 128^{3}, 320^{3}$ \\
64 & $\left\{48^{3}, 64^{3}\right\}, 96^{3}, 128^{3}, 320^{3}$ \\
80 & $\left\{64^{3}\right\}, 128^{3}, 320^{3}$ \\
{$[100$} & $\left.128^{3}, 192^{3}, 320^{3}\right]$ \\
{$[140$} & $\left.\left\{128^{3}\right\}, 192^{3}, 320^{3}\right]$ \\
{$[180$} & $\left.\left\{192^{3}\right\}, 320^{3}\right]$ \\
\hline \hline
\end{tabular}

Table 1: The lattice spacings (parametrised by $\beta$, cf. Eq. (2.7)) and the volumes (in lattice units, $N^{3}$, so that $V=N^{3} a^{3}$ ) studied. On each lattice we have collected $\sim 10^{4} \ldots 10^{6}$ independent measurements. The lattices in curly brackets have been left out from the infinite-volume extrapolations, while for the lattices in square brackets the significance loss due to the ultraviolet subtractions in Eq. (2.12) is so large (six orders of magnitude or more) that the subtracted values have little effect on our final fit (see below).

\section{Lattice measurements}

The goal of the numerical study is to measure the plaquette expectation value, $\left\langle 1-\frac{1}{3} \operatorname{Tr}\left[P_{12}\right]\right\rangle_{a}$, as a function of $\beta$, such that the extrapolation in Eq. (2.12) can be carried out. For each $\beta$, the infinite-volume limit needs to be taken. Given that the theory has a mass gap, we expect that finite-volume effects are exponentially small, if the length of the box $L$ is large compared with the confinement scale, $\sim 1 / g_{3}^{2}$. Writing $L=N a$, where $N$ is the number of lattice sites, the requirement $L \gg 1 / g_{3}^{2}$ converts to $\beta / N \ll 6$ (cf. Eq. (2.17)). Detailed studies with other observables show that in practice the finite-volume effects are invisible as soon as $\beta / N<1$ [20]. The values of $\beta$ and $N$ that we have employed are shown in Table 11 Earlier lattice measurements of the same observable were carried out with a volume $N^{3}=32^{3}$, with values of $\beta$ up to $\beta=30$ [21].

It is important to stress that the subtractions in Eq. (2.12) lead to a major significance loss. Essentially, we need to evaluate numerically the fourth derivative with respect to $\beta^{-1}$ of the function $\left\langle 1-\frac{1}{3} \operatorname{Tr}\left[P_{12}\right]\right\rangle_{a}$, at the point $\beta^{-1}=0$. Another way to express the problem is that as the numbers $c_{1}, \ldots, c_{4}$ are of order unity (cf. Eqs. (2.13)-(2.17)), the dominant term, $c_{1} / \beta$, is about six orders of magnitude larger than the effect we are interested in, $\sim 1 / \beta^{4}$, if $\beta \sim 100$. Therefore the relative error of our lattice measurements should be smaller than one 


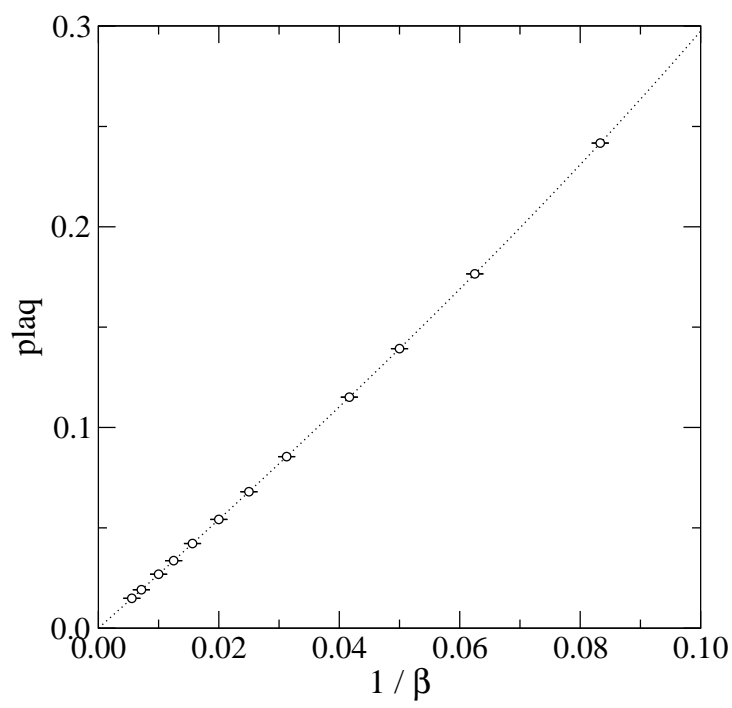

Figure 1: The plaquette expectation value, "plaq" $\equiv\left\langle 1-\frac{1}{3} \operatorname{Tr}\left[P_{12}\right]\right\rangle_{a}$, as a function of $1 / \beta$. Statistical errors are (much) smaller than the symbol sizes. The dotted curve contains the four known terms $c_{1} / \beta+c_{2} / \beta^{2}+c_{3} / \beta^{3}+c_{4} \ln \beta / \beta^{4}$ from Eq. (2.12), together with terms of the type $1 / \beta^{4}, 1 / \beta^{5}$ and $1 / \beta^{6}$ with fitted coefficients.

part in a million. We also need to know the coefficients $c_{i}$ with good precision.

Lattice-measured values of $\left\langle 1-\frac{1}{3} \operatorname{Tr}\left[P_{12}\right]\right\rangle_{a}$ are shown in Fig. 1] as a function of $1 / \beta$. In order to demonstrate the accuracy requirements we are faced with, Fig. 2 shows $\beta^{4}\langle 1-$ $\left.\frac{1}{3} \operatorname{Tr}\left[P_{12}\right]\right\rangle_{a}$, before and after the various subtractions. It is observed from Fig. 2 that after all the subtractions, this function indeed appears to have a finite limit for $\beta \rightarrow \infty$, or $1 / \beta \rightarrow 0$.

For each $\beta$, we have carried out simulations at a number of different lattice extents $N$; examples are shown in Fig. 3. No significant volume dependence is observed for $\beta / N<1$, and we thus estimate the infinite-volume limit by fitting a constant to data in this range.

Given the infinite-volume estimates, we extrapolate the data to the continuum limit, $\beta \rightarrow$ $\infty$. In Fig. 4 we show the functions $\beta^{4}\left\{\left\langle 1-\frac{1}{3} \operatorname{Tr}\left[P_{12}\right]\right\rangle_{a}-\left[c_{1} / \beta+c_{2} / \beta^{2}+c_{3} / \beta^{3}\right]\right\}$ and $\beta^{4}\left\{\left\langle 1-\frac{1}{3} \operatorname{Tr}\left[P_{12}\right]\right\rangle_{a}-\left[c_{1} / \beta+c_{2} / \beta^{2}+c_{3} / \beta^{3}+c_{4} \ln \beta / \beta^{4}\right]\right\}$. It is observed how even the 4-loop logarithmic divergence is visible in the data, as some upwards curvature for $1 / \beta \lesssim 0.06$. On the other hand, for $1 / \beta \leq 0.01$ the significance loss due to the subtractions grows rapidly and the error bars become quite large, so that these data points have little effect on the fit.

The continuum extrapolation is carried out by fitting a function $d_{1}+d_{2} / \beta+d_{3} / \beta^{2}$ to the infinite-volume extrapolated data for $\beta^{4}\left\{\left\langle 1-\frac{1}{3} \operatorname{Tr}\left[P_{12}\right]\right\rangle_{a}-\left[c_{1} / \beta+c_{2} / \beta^{2}+c_{3} / \beta^{3}+c_{4} \ln \beta / \beta^{4}\right]\right\}$, in the range $0.01<1 / \beta<0.10$. We find that this functional form describes the data very well. The fitted values are $d_{1}=19.4 \ldots 20.7, d_{2}=110 \ldots 63, d_{3}=717 \ldots 1101$, with $\chi^{2} /$ dof $=5.8 / 6$, where the intervals indicate the projections of the $68 \%$ confidence level contour (i.e. the 


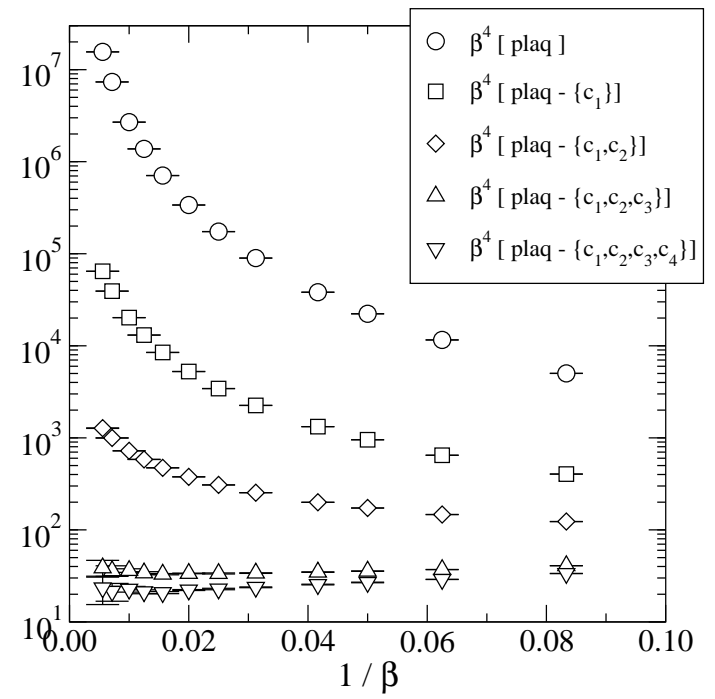

Figure 2: The significance loss due to the subtractions of the various ultraviolet divergent contributions in the gluon condensate. Here again "plaq" $\equiv\left\langle 1-\frac{1}{3} \operatorname{Tr}\left[P_{12}\right]\right\rangle_{a}$, and the symbols $c_{i}$ in the curly brackets indicate which subtractions of Eq. (2.12) have been taken into account.

surface where $\left.\chi^{2}=\chi_{\min }^{2}+3.53\right)$ onto the various axes, from one end of the elongated ellipse to the other. ${ }^{2}$ We have also estimated the systematic errors from the effect of higher order terms in the fit ansatz, and found that they are of the same order as these intervals, which we thus consider as our combined error estimates. Returning back to Eq. (2.12), we then obtain our final result,

$$
B_{\mathrm{G}}+\left(\frac{43}{12}-\frac{157}{768} \pi^{2}\right) c_{4}^{\prime}=\left(\frac{2 \pi^{2}}{27}\right)^{2} \times(20.0 \pm 0.7)=10.7 \pm 0.4
$$

where we have inserted $N_{\mathrm{c}}=3$.

\section{Conclusions}

The purpose of this paper has been to study the expectation value of the elementary plaquette in pure $\mathrm{SU}(3)$ lattice gauge theory in three dimensions, as well as to outline how the $\overline{\mathrm{MS}}$ scheme gluon condensate of the continuum theory can be extracted from it. To achieve this goal, we have carried out high precision numerical Monte Carlo simulations close to the continuum limit, corresponding to lattice spacings $0.05 \lesssim a g_{3}^{2} \lesssim 0.5$, where $g_{3}^{2}$ is the gauge coupling.

\footnotetext{
${ }^{2}$ If the three largest $\beta$ 's are included in the fit, the parameters remain essentially the same, $d_{1}=19.4 \ldots 20.8$, $d_{2}=107 \ldots 62, d_{3}=733 \ldots 1117$, while $\chi^{2} /$ dof $=7.0 / 9$ has decreased due to the large error bars at these $\beta$ 's.
} 

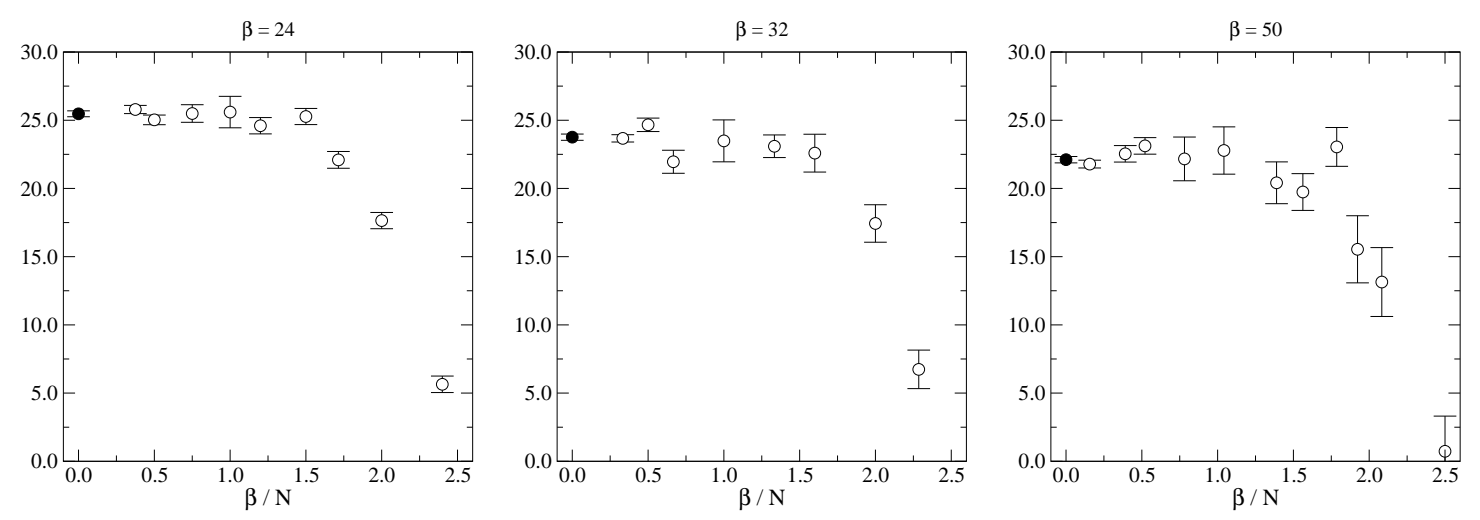

Figure 3: Finite-volume values for $\beta^{4}\left\{\left\langle 1-\frac{1}{3} \operatorname{Tr}\left[P_{12}\right]\right\rangle_{a}-\left[c_{1} / \beta+c_{2} / \beta^{2}+c_{3} / \beta^{3}+c_{4} \ln \beta / \beta^{4}\right]\right\}$, as a function of the physical extent $\beta / N=6 / g_{3}^{2} L$ of the box. The solid symbols indicate the infinite-volume estimates, obtained by fitting a constant to data in the range $\beta / N<1$.

When the leading perturbative terms, up to 4-loop level, are subtracted from the plaquette expectation value, and the result is divided by $\left(a g_{3}^{2}\right)^{4}$, a finite quantity remains (the right-hand side of Eq. (2.12), without $c_{4}^{\prime}$ ) which can be taken as the definition of a renormalised gluon condensate in lattice regularization (in certain units). We have carried out the subtractions and the extrapolation $a g_{3}^{2} \rightarrow 0$, and shown that our data appear to be precise enough to determine the remainder with less than $5 \%$ errors, cf. Fig. 4 and Eq. (3.1).

To relate this number to the gluon condensate in some continuum scheme, say $\overline{\mathrm{MS}}$, a further perturbative 4-loop matching computation remains to be completed, fixing the constant $c_{4}^{\prime}$ in Eqs. (2.12), (3.11). Our study should provide a strong incentive for finalising this challenging but feasible task, and there indeed is work in progress with this goal. The $\overline{\mathrm{MS}}$ scheme conversion is also needed in order to apply our result in the context of finite temperature physics, particularly for determining the $\mathcal{O}\left(g^{6} T^{4}\right)$ contribution to the pressure of hot QCD, since the other parts of that computation have been formulated in the $\overline{\mathrm{MS}}$ scheme [12].

\section{Acknowledgements}

We are grateful to H. Panagopoulos and A. Tsapalis for disclosing the results of Ref. [17] prior to publication. This work was partly supported by the Academy of Finland, contracts no. 77744 , 80170, and 104382, as well as by the Magnus Ehrnrooth Foundation. Simulations were carried out at the Finnish Center for Scientific Computing (CSC); the total amount of computing power used was about $4.5 \times 10^{16}$ flop. 


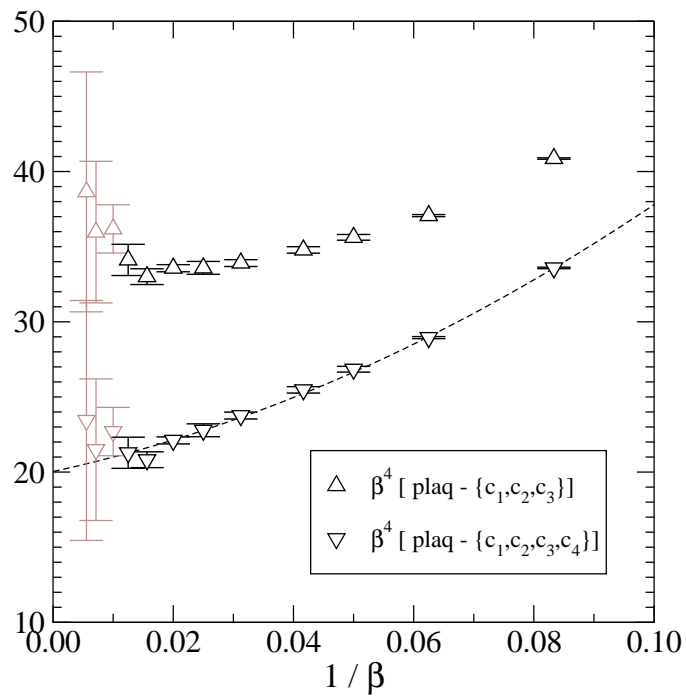

Figure 4: The infinite-volume extrapolated data, plotted as in Fig. 2. The effect of the 4-loop logarithmic divergence is to cause additional upwards "curvature" in the upper data set. The lower set includes all the subtractions, and should thus have a finite continuum limit. The continuum extrapolation (as described in the text) is indicated with the dashed line. The gray points have error bars so large that they are insignificant as far as the fit is concerned.

\section{References}

[1] K.G. Wilson, Phys. Rev. 179 (1969) 1499.

[2] M.B. Voloshin, Nucl. Phys. B 154 (1979) 365; Sov. J. Nucl. Phys. 36 (1982) 143 [Yad. Fiz. 36 (1982) 247]; H. Leutwyler, Phys. Lett. B 98 (1981) 447.

[3] A. Hasenfratz and P. Hasenfratz, Phys. Lett. B 93 (1980) 165; R.F. Dashen and D.J. Gross, Phys. Rev. D 23 (1981) 2340.

[4] K. Farakos, K. Kajantie, K. Rummukainen and M. Shaposhnikov, Nucl. Phys. B 442 (1995) 317 hep-lat/9412091.

[5] A.D. Linde, Phys. Lett. B 96 (1980) 289.

[6] D.J. Gross, R.D. Pisarski and L.G. Yaffe, Rev. Mod. Phys. 53 (1981) 43.

[7] P. Ginsparg, Nucl. Phys. B 170 (1980) 388; T. Appelquist and R.D. Pisarski, Phys. Rev. D 23 (1981) 2305.

[8] K. Kajantie, M. Laine, K. Rummukainen and M. Shaposhnikov, Nucl. Phys. B 458 (1996) 90 hep-ph/9508379. 
[9] E. Braaten and A. Nieto, Phys. Rev. D 53 (1996) 3421 hep-ph/9510408.

[10] K. Kajantie, M. Laine, K. Rummukainen and Y. Schröder, JHEP 04 (2003) 036 hep-ph/0304048.

[11] Y. Schröder, Nucl. Phys. B (Proc. Suppl.) 129 (2004) 572 hep-lat/0309112; in preparation.

[12] K. Kajantie, M. Laine, K. Rummukainen and Y. Schröder, Phys. Rev. D 67 (2003) 105008 hep-ph/0211321.

[13] A. Di Giacomo and G.C. Rossi, Phys. Lett. B 100 (1981) 481.

[14] U.M. Heller and F. Karsch, Nucl. Phys. B 251 (1985) 254.

[15] M. Laine and A. Rajantie, Nucl. Phys. B 513 (1998) 471 hep-lat/9705003.

[16] B. Allés, M. Campostrini, A. Feo and H. Panagopoulos, Phys. Lett. B 324 (1994) 433 hep-lat/9306001; B. Allés, A. Feo and H. Panagopoulos, Phys. Lett. B 426 (1998) 361 hep-lat/9801003; ibid. B 553 (2003) 337 (Erratum).

[17] H. Panagopoulos and A. Tsapalis, in preparation.

[18] F. Di Renzo, A. Mantovi, V. Miccio and Y. Schröder, JHEP 05 (2004) 006 hep-lat/0404003.

[19] F. Di Renzo, E. Onofri, G. Marchesini and P. Marenzoni, Nucl. Phys. B 426 (1994) 675 hep-lat/9405019.

[20] M.J. Teper, Phys. Rev. D 59 (1999) 014512 hep-lat/9804008; M. Laine and O. Philipsen, Phys. Lett. B 459 (1999) 259 hep-lat/9905004.

[21] F. Karsch, M. Lütgemeier, A. Patkós and J. Rank, Phys. Lett. B 390 (1997) 275 hep-lat/9605031. 